\title{
Canagliflozin for treatment of post-bariatric surgery hypoglycaemia unresponsive to other therapy
}

Dr Kamal Abouglila, Dr Kerri Devine, Department of Endocrinology, University Hospital North Durham

\section{BACKGROUND}

Reactive hypoglycaemia after gastric bypass surgery is characterized primarily by excessive postprandial hyperinsulinaemia and resultant neuroglycopaenic symptoms. The elusive pathophysiology of this problem has made treatment challenging. We describe the first case report where treatment with the Sodium Glucose Transporter Inhibitor canagliflozin has reduced hypoglycaemic episodes after failure of other therapies.

\section{CASE STUDY}

A 56 year old female with a history of obesity and diet controlled Type 2 Diabetes Mellitus underwent Roux-en-Y gastric bypass in 2011, resulting in weight loss of over 20 kilograms. Three years later she began experiencing almost daily episodes of symptomatic hypoglycaemia. Plasma glucose fell to $2.1 \mathrm{mmol} / \mathrm{L}$ postprandially without suppression of insulin $(39.8 \mathrm{mU} / \mathrm{L})$ or $C$ peptide $(6.25 \mathrm{nmol} / \mathrm{L})$. Flash glucose monitoring with the Free Style Libre device confirmed postprandial spikes of hyperglycaemia with subsequent hypoglycaemia.

MR imaging of the pancreas was normal, and anti-insulin antibodies and sulfonylurea screen were negative. Renal and liver disease and hypoadrenalism were excluded.

Dietary adjustments were advised and metformin commenced, with minimal improvement. Acarbose and octreotide were poorly tolerated due to gastrointestinal side effects.

A trial of canagliflozin $100 \mathrm{mg}$ daily was offered to the patient before contemplating surgical reversal. She experienced immediate remarkable symptomatic improvement associated with reduction of both hyperglycaemia and hypoglycaemia, documented on both capillary and flash glucose monitoring. This was sustained over a 9 month follow up period, and was further confirmed by 1 week off and one week on canagliflozin.
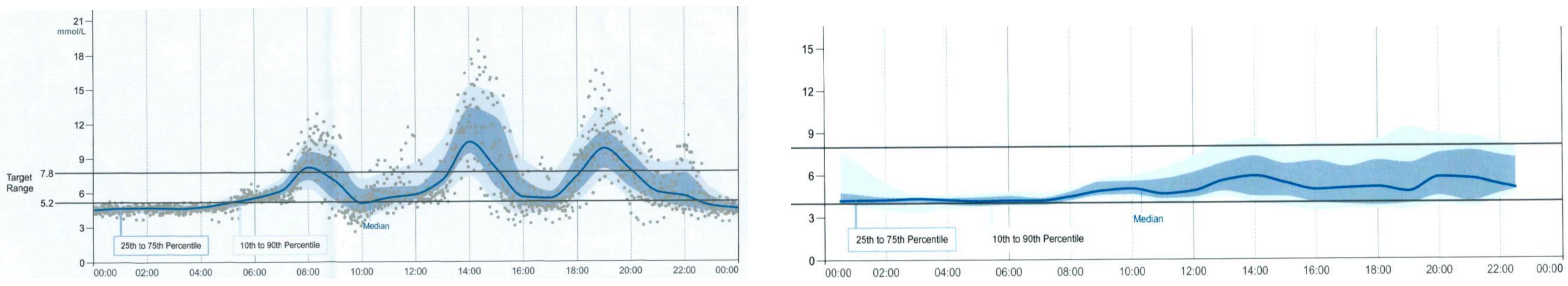

Fig 1. Pre and post treatment Freestyle Libre 14 day AGP (Ambulatory Glucose Profile) download showing interstitial glucose trends over a 24h period

\section{DISCUSSION}

Postprandial hypoglycemia has been successfully treated with an SGLT2 inhibitor in this gastric bypass patient with impaired glucose tolerance after failed response to diet, metformin, acarbose and octreotide. Canagliflozin has helped to both reduce the frequency of hypoglycaemia and the degree of blood glucose excursion after carbohydrate. This case also demonstrates the usefulness of interstitial glucose monitoring in revealing blood glucose trends and response to treatment in such patients. 